\title{
New integral inequalities of Hermite-Hadamard's and Simpson's type for twice differentiable mappings
}

\author{
Zaffer Elahi $^{1} \cdot$ Muhammad Muddassar $^{1}$
}

Received: 4 April 2019 / Accepted: 22 August 2019 / Published online: 4 September 2019

(c) The Author(s) 2019

\begin{abstract}
In this paper, some new interesting results based on quasi-geometrically convex mappings of both Hadamard's and Simpson's inequalities have been constructed defining a new identity for twice differentiable mappings.
\end{abstract}

Keywords Quasi-geometrically convex functions · Hermite-Hadamard-type inequalities · Simpson-type inequality

Mathematics Subject Classification $26 \mathrm{D} 10 \cdot 26 \mathrm{D} 15 \cdot 39 \mathrm{~A} 12$

\section{Introduction and preliminary results}

Any function $f$ be continuous on an interval $I$ such that its value at the midpoint of the interval does not exceed from the arithmetic mean of boundary values of the interval, termed as a convex function. The investigation of an important mathematical problem informs how function behaves using means. Jensen convex function is one of the eminent cases that deals with arithmetic mean [1, pp.2]. A function $f$ is convex on an interval $[p, q]$ if

$f(\alpha \eta+\beta \zeta) \leq \alpha f(\eta)+\beta f(\zeta)$,

where $\eta, \zeta \in[p, q]$ and $\alpha, \beta \in] 0,1]$. Further, a real-valued function defined on a non-empty subinterval $I$ of $\mathbb{R}$ is called convex if we replace $\alpha+\beta=1$ for all points $\eta, \zeta \in I$ in the above inequality. It is called strictly convex if the above inequality holds strictly whenever $\eta$ and $\zeta$ are distinct points. In addition, $-f$ is convex/ strictly convex; accordingly, $f$ is concave/ strictly concave. Further, a function is called affine if it is both convex and concave. The application of Hermite-Hadamard-type inequalities and convexities can be found in [2-7].

Zaffer Elahi

zafferelahi@gmail.com

Muhammad Muddassar

malik.muddassar@gmail.com

1 Department of Basic Sciences, University of Engineering and Technology, Taxila, Pakistan
In the field of applied sciences, the occurrence of new mathematical inequalities puts the foundation for the heuristic algorithms. Hermite-Hadamard's is one of the main inequalities that yields explicitly the error bounds of the trapezoidal and midpoint rules for a smooth convex function $f:[p, q] \rightarrow \mathbb{R}$, defined as

$f\left(\frac{p+q}{2}\right) \leq \frac{1}{q-p} \int_{p}^{q} f(\tau) d \tau \leq \frac{f(p)+f(q)}{2}$.

For $f$ to be concave, these inequalities also hold in reverse order. More precisely, Hermite-Hadamard's inequality (1.1) may depict the concept of convexity and follows from Jensen inequality. Inequality (1.1) has gained much attention among researchers due to its remarkable characteristics in refinements and generalizations, as well. Simpson's is another well-known type of inequality, defined as

$$
\begin{aligned}
\mid \frac{1}{3}\left[2 f\left(\frac{p+q}{2}\right)+\frac{f(p)+f(q)}{2}\right] \\
\quad-\frac{1}{q-p} \int_{p}^{q} f(\eta) d \eta \mid \leq \frac{1}{2880}\left\|f^{(i v)}\right\|_{\infty}(q-p)^{4}
\end{aligned}
$$

where $f:[p, q] \rightarrow \mathbb{R}$ is a four times continuous differentiable mapping on $(p, q)$ and $\left\|f^{(i v)}\right\|_{\infty}=\sup _{\eta \in(p, q)}\left|f^{(i v)}(\eta)\right|<\infty$. It is to be mentioned that the classical Simpson quadrature formula cannot be applied for $f$ is neither differentiable four times nor $f^{(i v)}$ bounded on $(p, q)$.

Moreover, the quasi-convex function, $f:[p, q] \rightarrow \mathbb{R}$, is the generalization of convex function, defined as 
$f(\alpha \eta+\beta \zeta) \leq \sup \{f(\eta), f(\zeta)\}$,

where $\eta, \zeta \in[p, q]$ and $\alpha, \beta \in] 0,1]$ such that $\alpha+\beta=1$.

Initially, the notion of geometrically convex was introduced by Niculescu in $[8,9]$ and produced as

Definition 1 A function $f: I \subseteq \mathbb{R}^{+} \rightarrow \mathbb{R}^{+}$is said to be $G G$ convex (called geometrically convex function) if

$f\left(\eta^{\alpha} \zeta^{\beta}\right) \leq f^{\alpha}(\eta) f^{\beta}(\zeta)$

where $\eta, \zeta \in[p, q]$ and $\alpha, \beta \in] 0,1]$ such that $\alpha+\beta=1$.

Niculescu, in the same article, defined the term geometric - arithmatically convex with notation $G A$-convex, as

Definition 2 A function $f: I \subseteq \mathbb{R}^{+} \rightarrow \mathbb{R}$ is termed as $G A$ convex (called geometrically convex function) if

$f\left(\eta^{\alpha} \zeta^{\beta}\right) \leq \alpha f(\eta)+\beta f(\zeta)$,

where $\eta, \zeta \in[p, q]$ and $\alpha, \beta \in] 0,1]$ such that $\alpha+\beta=1$.

The following definition of quasi-geometrically convex functions was first reported by İşcan [10]

Definition 3 A function $f: I \subseteq \mathbb{R}^{+} \rightarrow \mathbb{R}$ is said to be quasiconvex if

$f\left(\eta^{\alpha} \zeta^{\beta}\right) \leq \sup \{f(\eta), f(\zeta)\}$,

where $\eta, \zeta \in[p, q]$ and $\alpha, \beta \in] 0,1]$ such that $\alpha+\beta=1$.

Both $G A$-convex and geometrically convex functions are quasi-geometrically convex functions, but there exist quasigeometrically convex functions which are neither $G A$-convex nor $G G$-convex discussed in $[11,12]$.

Recently, İşcan et al. [13] developed some results based on single differentiability for quasi-geometrically convex functions using the identity

Lemma 1 A function $f: I \subseteq \mathbb{R}^{+} \rightarrow \mathbb{R}$ be a differentiable function on $I^{o}$ such that $f \in L^{1}([p, q])$, where $p, q \in I$ with $p<q$. Then for all $\lambda, \mu \in \mathbb{R}$, we have

$$
\begin{aligned}
& I_{f}(\lambda, \mu, p, q)=\ln \left(\frac{q}{p}\right) \\
& \quad \times\left\{\int_{0}^{\frac{1}{2}}(\tau-\mu) p^{1-\tau} q^{\tau} f^{\prime}\left(p^{1-\tau} q^{\tau}\right) d \tau\right. \\
& \left.\quad+\int_{\frac{1}{2}}^{1}(\tau-\lambda) p^{1-\tau} q^{\tau} f^{\prime}\left(p^{1-\tau} q^{\tau}\right) d \tau\right\},
\end{aligned}
$$

where

$$
\begin{aligned}
& I_{f}(\lambda, \mu, p, q)=(\lambda-\mu) f(\sqrt{p q})+\mu f(p)+(1-\lambda) f(q) \\
& -\frac{1}{\ln \left(\frac{q}{p}\right)} \int_{p}^{q} \frac{f(\xi)}{\xi} d \xi .
\end{aligned}
$$

This paper is in the continuation of [13]. The main purpose of the paper is to develop some new integral inequalities of both Hadamard and Simpson type for twice differentiable mappings using new integral identity.

\section{Main results}

The following identity is needed to prove main results.

Lemma 2 A function $f: I \subseteq \mathbb{R}^{+} \rightarrow \mathbb{R}$ be a differentiable function on $I^{o}$ such that $f \in L^{1}([p, q])$, where $p, q \in I$ with $p<q$. Then for all $\lambda, \mu \in \mathbb{R}$, we have

$$
\begin{aligned}
& \left|M_{f}(\lambda, \mu, p, q)\right|=\left(\ln \left(\frac{q}{p}\right)\right)^{2} \\
& \quad \times\left\{\int_{0}^{\frac{1}{2}} \tau(\tau-\mu) p^{2(1-\tau)} q^{2 \tau} f^{\prime \prime}\left(p^{1-\tau} q^{\tau}\right) d \tau\right. \\
& \left.\quad+\int_{\frac{1}{2}}^{1}(1-\tau)(\tau-\lambda) p^{2(1-\tau)} q^{2 \tau} f^{\prime \prime}\left(p^{1-\tau} q^{\tau}\right) d \tau\right\},
\end{aligned}
$$

where

$$
\begin{aligned}
& M_{f}(\lambda, \mu, p, q)=(\lambda-\mu+1) f(\sqrt{p q}) \\
& \quad+\mu f(p)+(1-\lambda) f(q) \\
& \quad+\frac{\sqrt{p q}(\lambda+\mu-1)}{2} \ln \left(\frac{q}{p}\right) f^{\prime}(\sqrt{p q}) \\
& \quad-\frac{2}{\ln (q / p)} \int_{p}^{q} \frac{f(\xi)}{\xi} d \xi .
\end{aligned}
$$

Proof Using integration rules and changing parameter, it leads to the result.

Theorem 1 A function $f: I \subseteq \mathbb{R}^{+} \rightarrow \mathbb{R}$ be a twice differentiable function on $I^{o}$ such that $f^{\prime \prime} \in L^{1}([p, q])$, where $p, q \in I$ with $p<q$. If $\left|f^{\prime \prime}\right|^{k}$ is quasi-geometrically convex on $[p, q]$ for some fixed $k \geq 1$ and $0 \leq \mu \leq 1 / 2 \leq \lambda \leq 1$, then the following inequality holds 
Mathematical Sciences (2019) 13:279-285

281

$$
\begin{aligned}
& \left|M_{f}(\lambda, \mu, p, q)\right| \leq\left(\ln \left(\frac{q}{p}\right)\right) \\
& \quad \times\left(\sup \left\{\left|f^{\prime \prime}(p)\right|^{k},\left|f^{\prime \prime}(q)\right|^{k}\right\}\right)^{\frac{1}{k}}\left\{c_{1}^{1-\frac{1}{k}}(\mu) c_{3}^{\frac{1}{k}}(\mu, k, p, q)\right. \\
& \left.\quad+c_{2}^{1-\frac{1}{k}}(\lambda) c_{4}^{\frac{1}{k}}(\lambda, k, p, q)\right\},
\end{aligned}
$$

where

$$
\begin{aligned}
& c_{1}(\mu)=\frac{\mu^{3}}{3}-\frac{\mu}{8}+\frac{1}{24}, \\
& c_{2}(\lambda)=-\frac{\lambda^{3}}{3}+\lambda^{2}-\frac{7 \lambda}{8}+\frac{1}{4}, \\
& c_{3}(\mu, k, p, q)=\frac{1}{2 k\left(2 \ln \left(\frac{q}{p}\right)\right)} \\
& \times\left[8 \mu^{2} p^{2 k(1-\mu)} \mathrm{L}\left(p^{2 k \mu}, q^{2 k \mu}\right)-\frac{8 \mu p^{2 k(1-\mu)}}{k\left(2 \ln \left(\frac{q}{p}\right)\right)}\right. \\
& \times \mathrm{L}\left(p^{2 k \mu}, q^{2 k \mu}\right)+\frac{p^{k}}{k\left(\ln \left(\frac{q}{p}\right)\right)} \mathrm{L}\left(p^{k}, q^{k}\right) \\
& +\frac{10 \mu p^{2 k}}{k\left(2 \ln \left(\frac{q}{p}\right)\right)} \\
& +\frac{2(\mu-1)}{k\left(2 \ln \left(\frac{q}{p}\right)\right)}(p q)^{k}+\frac{1-2 \mu}{2}(p q)^{k},
\end{aligned}
$$

and

$$
\begin{aligned}
& c_{4}(\lambda, k, p, q)=\frac{1}{2 k\left(2 \ln \left(\frac{q}{p}\right)\right)} \\
& \times\left[\frac{2(1-\lambda) q^{2 k}}{k\left(2 \ln \left(\frac{q}{p}\right)\right)}-\frac{(2 \lambda-1)(p q)^{k}}{2}+4 \lambda(1-\lambda) p^{2 k(1-\lambda)}\right. \\
& \times \mathrm{L}\left(p^{2 k \lambda}, q^{2 k \lambda}\right)-2 \lambda p^{k} \mathrm{~L}\left(p^{k}, q^{k}\right) \\
& +\frac{8 p^{2 k(1-\lambda)} q^{2 k \lambda}}{k^{2}\left(2 \ln \left(\frac{q}{p}\right)\right)^{2}}+\frac{2 \lambda(p q)^{k}}{k\left(2 \ln \left(\frac{q}{p}\right)\right)} \\
& \left.-\frac{4 q^{2 k}}{k^{2}\left(2 \ln \left(\frac{q}{p}\right)\right)^{2}}-\frac{4(p q)^{k}}{k^{2}\left(2 \ln \left(\frac{q}{p}\right)\right)^{2}}\right] .
\end{aligned}
$$

Proof Since $\left|f^{\prime \prime}\right|^{k}$ is quasi-geometrically convex on $[p, q]$ for all $\tau \in[0,1]$

$$
\left|f^{\prime \prime}\left(p^{1-\tau} q^{\tau}\right)\right|^{k} \leq \sup \left\{\left|f^{\prime \prime}(p)\right|^{k},\left|f^{\prime \prime}(q)\right|^{k}\right\}
$$

Using Lemma 2 and power mean inequality, it yields

$$
\begin{aligned}
& \left|M_{f}(\lambda, \mu, p, q)\right|=\left(\ln \left(\frac{q}{p}\right)\right)^{2} \\
& \quad \times\left\{( \int _ { 0 } ^ { \frac { 1 } { 2 } } | \tau ( \tau - \mu ) | d \tau ) ^ { 1 - \frac { 1 } { k } } \left(\int_{0}^{\frac{1}{2}}|\tau(\tau-\mu)|\left(p^{2(1-\tau)} q^{2 \tau}\right)^{k}\right.\right. \\
& \left.\quad \times \sup \left\{\left|f^{\prime \prime}(p)\right|^{k},\left|f^{\prime \prime}(q)\right|^{k}\right\}\right)^{\frac{1}{k}} \\
& \quad+\left(\int_{\frac{1}{2}}^{1}|(1-\tau)(\tau-\lambda)| d \tau\right)^{1-\frac{1}{k}}\left(\int_{\frac{1}{2}}^{1}|(1-\tau)(\tau-\lambda)|\right. \\
& \left.\left.\quad \times\left(p^{2(1-\tau)} q^{2 \tau}\right)^{k} \sup \left\{\left|f^{\prime \prime}(p)\right|^{k},\left|f^{\prime \prime}(q)\right|^{k}\right\}\right)^{\frac{1}{k}}\right\} .
\end{aligned}
$$

Here we assume that

$$
\begin{aligned}
& c_{1}(\mu)=\int_{0}^{\frac{1}{2}}|\tau(\tau-\mu)| d \tau=\int_{0}^{\mu}(\tau(\mu-\tau)) d \tau \\
&+\int_{\mu}^{\frac{1}{2}}(\tau(\tau-\mu)) d \tau=\frac{\mu^{3}}{3}-\frac{\mu}{8}+\frac{1}{24}, \\
& c_{2}(\lambda)=\int_{\frac{1}{2}}^{1}|(1-\tau)(\tau-\lambda)| d \tau=\int_{\frac{1}{2}}^{\lambda}(1-\tau)(\lambda-\tau) d \tau \\
&+\int_{\lambda}^{1}(1-\tau)(\tau-\lambda) d \tau=-\frac{\lambda^{3}}{3} \\
&+\lambda^{2}-\frac{7 \lambda}{8}+\frac{1}{4}, \\
& c_{3}(\mu, k, p, q)=\int_{0}^{\frac{1}{2}}|\tau(\mu-\tau)|\left(p^{2(1-\tau)} q^{2 \tau}\right)^{k} d \tau \\
&=\int_{0}^{\mu} \tau(\mu-\tau)\left(p^{2(1-\tau)} q^{2 \tau}\right)^{k} d \tau \\
& \quad+\int_{\mu}^{\frac{1}{2}} \tau(\tau-\mu)\left(a^{2(1-\tau)} q^{2 \tau}\right)^{k} d \tau .
\end{aligned}
$$

Using substitution $\xi=p^{2(1-\tau)} q^{2 \tau}$ in $c_{3}(\mu, k, p, q)$, it leads to

Springer 


$$
\begin{aligned}
& \int_{0}^{\mu} \tau(\mu-\tau)\left(p^{2(1-\tau)} q^{2 \tau}\right) d \tau \\
& =\frac{\mu}{\left(2 \ln \left(\frac{q}{p}\right)\right)^{2}} \int_{p^{2}}^{p^{2(1-\mu)} q^{2 \mu}} \xi^{k-1} \ln \left(\frac{\xi}{p^{2}}\right) d \xi \\
& -\frac{\mu}{\left(2 \ln \left(\frac{q}{p}\right)\right)^{3}} \int_{p^{2}}^{p^{2(1-\mu)} q^{2 \mu}} \xi^{k-1} \ln \left(\frac{\xi}{p^{2}}\right) d \xi \\
& \int_{0}^{\mu} \tau(\mu-\tau)\left(p^{2(1-\tau)} q^{2 \tau}\right) d \tau \\
& =\frac{\mu p^{2 k(1-\mu)} q^{2 k \mu}}{k^{2}\left(2 \ln \left(\frac{q}{p}\right)\right)^{2}}-\frac{2 p^{2 k(1-\mu)} q^{2 k \mu}}{k^{3}\left(2 \ln \left(\frac{q}{p}\right)\right)^{3}} \\
& +\frac{\mu p^{2 k}}{k^{2}\left(2 \ln \left(\frac{q}{p}\right)\right)^{2}}+\frac{\mu p^{2 k}}{k^{3}\left(2 \ln \left(\frac{q}{p}\right)\right)^{3}} \\
& \int_{\mu}^{\frac{1}{2}} \tau(\tau-\mu)\left(p^{2(1-t)} q^{2 \tau}\right) d \tau \\
& =\frac{1}{\left(2 \ln \left(\frac{q}{p}\right)\right)^{3}} \int_{p^{2(1-\mu)} q^{2 \mu}}^{p q} \xi^{k-1}\left(\ln \left(\frac{\xi}{p^{2}}\right)\right)^{2} d \xi \\
& -\frac{\mu}{\left(2 \ln \left(\frac{q}{p}\right)\right)^{2}} \int_{p^{2(1-\mu)} q^{2 \mu}}^{p q} \xi^{k-1} \ln \left(\frac{\xi}{p^{2}}\right) d \xi \\
& \int_{\mu}^{\frac{1}{2}} \tau(\tau-\mu)\left(p^{2(1-\tau)} q^{2 \tau}\right) d \tau \\
& =\frac{(1-2 \mu)(p q)^{k}}{8 k \ln \left(\frac{q}{p}\right)}+\frac{(\mu-1)(p q)^{k}}{k^{2}\left(2 \ln \left(\frac{q}{p}\right)\right)^{2}}+\frac{3 \mu p^{2 k(1-\mu)} q^{2 k \xi}}{k^{2}\left(2 \ln \left(\frac{q}{p}\right)\right)^{2}} \\
& +\frac{2(p q)^{k}}{k^{3}\left(2 \ln \left(\frac{q}{p}\right)\right)^{3}}-\frac{2 p^{2 k(1-\mu)} q^{2 k \mu}}{k^{3}\left(2 \ln \left(\frac{p}{q}\right)\right)^{3}} \text {. }
\end{aligned}
$$

Finally, we get

$$
\begin{aligned}
& c_{3}(\mu, k, p, q)=\frac{1}{2 k\left(2 \ln \left(\frac{q}{p}\right)\right)} \\
& \times\left[8 \mu^{2} p^{2 k(1-\mu)} \mathrm{L}\left(p^{2 k \mu}, q^{2 k \mu}\right)-\frac{8 \mu p^{2 k(1-\mu)}}{k\left(2 \ln \left(\frac{q}{p}\right)\right)}\right. \\
& \times \mathrm{L}\left(p^{2 k \mu}, q^{2 k \mu}\right)+\frac{p^{k}}{k\left(\ln \left(\frac{q}{p}\right)\right)} \mathrm{L}\left(p^{k}, q^{k}\right)+\frac{10 \mu p^{2 k}}{k\left(2 \ln \left(\frac{q}{p}\right)\right)} \\
& \left.+\frac{2(\mu-1)}{k\left(2 \ln \left(\frac{q}{p}\right)\right)}(p q)^{k}+\frac{1-2 \mu}{2}(p q)^{k}\right] .
\end{aligned}
$$

And

$$
\begin{aligned}
c_{4}(\lambda, k, p, q) & =\int_{\frac{1}{2}}^{1}|(1-\tau)(\tau-\lambda)|\left(p^{2(1-\tau)} q^{2 \tau}\right)^{k} d \tau \\
& =\int_{\frac{1}{2}}^{\lambda} \tau(\mu-\tau)\left(p^{2(1-\tau)} q^{2 \tau}\right)^{k} d \tau \\
& +\int_{\lambda}^{1} \tau(\tau-\mu)\left(p^{2(1-\tau)} q^{2 \tau}\right)^{k} d \tau .
\end{aligned}
$$

Using same substitution $\xi=p^{2(1-\tau)} q^{2 \tau}$ in $c_{4}(\lambda, k, p, q)$, we have

$$
\begin{aligned}
& c_{4}(\lambda, k, p, q)=\frac{1}{2 k\left(2 \ln \left(\frac{q}{p}\right)\right)} \\
& \times\left[\frac{2(1-\lambda) q^{2 k}}{k\left(2 \ln \left(\frac{q}{p}\right)\right)}-\frac{(2 \lambda-1)(p q)^{k}}{2}+4 \lambda(1-\lambda) p^{2 k(1-\lambda)}\right. \\
& \times \mathrm{L}\left(p^{2 k \lambda}, q^{2 k \lambda}\right)-2 \lambda p^{k} \mathrm{~L}\left(p^{k}, q^{k}\right) \\
& +\frac{8 p^{2 k(1-\lambda) q^{2 k \lambda}}}{k^{2}\left(2 \ln \left(\frac{q}{p}\right)\right)^{2}}+\frac{2 \lambda(p q)^{k}}{k\left(2 \ln \left(\frac{q}{p}\right)\right)} \\
& \left.-\frac{4 q^{2 k}}{k^{2}\left(2 \ln \left(\frac{q}{p}\right)\right)^{2}}-\frac{4(p q)^{k}}{k^{2}\left(2 \ln \left(\frac{q}{p}\right)\right)^{2}}\right] .
\end{aligned}
$$

This completes the proof.

Corollary 1 A function $f: I \subseteq \mathbb{R}^{+} \rightarrow \mathbb{R}$ be a twice differentiable function on $I^{o}$ such that $f^{\prime \prime} \in L^{1}([p, q])$, where $p, q \in I$ with $p<q$. If $\left|f^{\prime \prime}\right|^{k}$ is quasi-geometrically convex on $[p, q]$ for some fixed $k \geq 1$ and for $l, m \in \mathbb{R}$ with $l<m$, then the following inequality holds

$$
\begin{aligned}
\mid m_{f} & \left(\frac{l}{m}, p, q\right) \mid \leq \frac{8 l^{3}-3 m^{2} l+m^{3}}{24 m^{3}}\left(\ln \left(\frac{q}{p}\right)\right) \\
& \times\left(\sup \left\{\left|f^{\prime \prime}(p)\right|^{k},\left|f^{\prime \prime}(q)\right|^{k}\right\}\right)^{\frac{1}{k}} \\
& \times\left\{c_{3}^{\frac{1}{q}}\left(\frac{l}{m}, k, p, q\right)+c_{4}^{\frac{1}{k}}\left(\frac{l}{m}, k, p, q\right)\right\},
\end{aligned}
$$

where

$$
\begin{aligned}
& m_{f}\left(\frac{l}{m}, p, q\right)=2\left(\frac{m-l}{m}\right) f(\sqrt{p q}) \\
& +\frac{l}{m}(f(p)+f(q))-\frac{2}{\ln \left(\frac{q}{p}\right)} \int_{p}^{q} \frac{f(\xi)}{\xi} d \xi
\end{aligned}
$$


Mathematical Sciences (2019) 13:279-285

283

and

$$
\begin{aligned}
& c_{1}\left(\frac{l}{m}\right)=c_{2}\left(\frac{l}{m}\right)=\frac{8 l^{3}-3 m^{2} l+m^{3}}{24 m^{3}}, \\
& c_{3}\left(\frac{l}{m}, k, p, q\right)=\frac{1}{2 k\left(2 \ln \left(\frac{q}{p}\right)\right)} \\
& \quad \times\left[\frac{8 l}{m} p^{\frac{2 k(m-l)}{m}} \frac{l k\left(2 \ln \left(\frac{q}{p}\right)\right)-m}{m q\left(2 \ln \left(\frac{q}{p}\right)\right)}\right) \mathrm{L}\left(p^{2 k \frac{l}{m}}, q^{2 k \frac{l}{m}}\right) \\
& +\frac{p^{k}}{k\left(\ln \left(\frac{q}{p}\right)\right)} \mathrm{L}\left(p^{k}, q^{k}\right)+\frac{10 l p^{2 k}}{m q\left(2 \ln \left(\frac{q}{p}\right)\right)} \\
& \left.\quad-\frac{2(m-l)}{m k\left(2 \ln \left(\frac{q}{p}\right)\right)}(p q)^{k}+\frac{(m-2 l)}{2 m}(p q)^{k}\right], \\
& c_{4}\left(\frac{l}{m}, k, p, q\right)=\frac{1}{2 k\left(2 \ln \left(\frac{q}{p}\right)\right)} \\
& \quad \times\left[\frac{8 l}{m} b^{\frac{2 k(m-l)}{m}} \frac{l k\left(2 \ln \left(\frac{q}{p}\right)\right)-m}{m k\left(2 \ln \left(\frac{q}{p}\right)\right)}\right] \mathrm{L}\left(p^{2 k \frac{l}{m}}, q^{2 k \frac{l}{m}}\right) \\
& +\frac{q^{k}}{k\left(\ln \left(\frac{q}{p}\right)\right)} \mathrm{L}\left(p^{k}, q^{k}\right)+\frac{10 l q^{2 k}}{m k\left(2 \ln \left(\frac{q}{p}\right)\right)} \\
& \left.\quad \frac{2(m-l)}{m k\left(2 \ln \left(\frac{q}{p}\right)\right)}(p q)^{k}+\frac{(m-2 l)}{2 m}(p q)^{k}\right] .
\end{aligned}
$$

Proof Proof is exchangeable with Theorem 1 using substituion $\lambda=1-\frac{l}{m}$ and $\mu=\frac{l}{m}$.

Theorem 2 A function $f: I \subseteq \mathbb{R}^{+} \rightarrow \mathbb{R}$ be a twice differentable function on $I^{o}$ such that $f^{\prime \prime} \in L^{1}([p, q])$, where $p, q \in I$ with $p<q$. If $\left|f^{\prime \prime}\right|^{k}$ is quasi-geometrically convex on $[p, q]$ for some fixed conjugate numbers $j, k \geq 0$ where $k>1$ and $0 \leq \mu \leq 1 / 2 \leq \lambda \leq 1$, then the following inequality holds

$$
\begin{aligned}
\left|M_{f}(\lambda, \mu, p, q)\right| \leq\left(\ln \left(\frac{q}{p}\right)\right)^{2} \\
\quad \times\left(\sup \left\{\left|f^{\prime \prime}(p)\right|^{k},\left|f^{\prime \prime}(q)\right|^{k}\right\}\right)^{\frac{1}{k}} \\
\quad \times\left\{c_{5}^{\frac{1}{j}}(j, \mu) c_{7}^{\frac{1}{k}}(k, p, q)+c_{6}^{\frac{1}{j}}(j, \lambda) c_{8}^{\frac{1}{k}}(k, p, q)\right\},
\end{aligned}
$$

where

$$
\begin{gathered}
c_{5}(j, \mu)=\frac{(1-2 \mu)^{j+1}}{4^{j+1}(j+1)(1-\mu)}, c_{6}(j, \lambda)=\frac{(2 \lambda-1)^{j+1}}{4^{j+1}(j+1) \lambda}, \\
c_{7}(k, p, q)=\frac{p^{k}}{2} \mathrm{~L}\left(p^{k}, q^{k}\right), c_{8}(k, p, q)=\frac{q^{k}}{2} \mathrm{~L}\left(p^{k}, q^{k}\right) .
\end{gathered}
$$

Proof From Lemma 2 by applying Hölder inequality and using the quasi-geometrically convexity on $[p, q]$ of $\left|f^{\prime \prime}\right|^{k}$, we have

$$
\begin{aligned}
& \left|M_{f}(\lambda, \mu, p, q)\right|=\left(\ln \left(\frac{q}{p}\right)\right)^{2} \\
& \quad \times\left\{( \int _ { 0 } ^ { \frac { 1 } { 2 } } | \tau ( \mu - \tau ) | ^ { j } d \tau ) ^ { \frac { 1 } { j } } \left(\int_{0}^{\frac{1}{2}}\left(p^{2(1-\tau)} q^{2 \tau}\right)^{k}\right.\right. \\
& \left.\quad \times \sup \left\{\left|f^{\prime \prime}(p)\right|^{k},\left|f^{\prime \prime}(q)\right|^{k}\right\} d \tau\right)^{\frac{1}{k}} \\
& \quad+\left(\int_{\frac{1}{2}}^{1}|(1-\tau)(\tau-\lambda)|^{j} d \tau\right)^{\frac{1}{j}}\left(\int_{\frac{1}{2}}^{1}\left(j^{2(1-\tau)} q^{2 \tau}\right)^{k}\right. \\
& \left.\left.\quad \times \sup \left\{\left|f^{\prime \prime}(p)\right|^{k},\left|f^{\prime \prime}(q)\right|^{k}\right\} d \tau\right)^{\frac{1}{k}}\right\} \\
& \left|M_{f}(\lambda, \mu, p, q)\right|=\left(\ln \left(\frac{q}{p}\right)\right)^{2} \\
& \quad \times\left(\sup \left\{\left|f^{\prime \prime}(p)\right|^{k},\left|f^{\prime \prime}(q)\right|^{q}\right\}\right)^{\frac{1}{k}}\left\{\left(\int_{0}^{\frac{1}{2}}|\tau(\mu-\tau)|^{j} d \tau\right)^{\frac{1}{j}}\right. \\
& \left.\quad+\left(\int_{\frac{1}{2}}^{1}|(1-\tau)(\tau-\lambda)|^{j} d \tau\right)^{\frac{1}{2}}\left(\int_{\frac{1}{2}}^{1}\left(p^{2(1-\tau)} k^{2 \tau}\right)^{k} d \tau\right)^{\frac{1}{k}}\right\} \\
& \left.\quad\left(p_{0}^{2(1-\tau)} q^{2 \tau}\right)^{k} d \tau\right)^{\frac{1}{k}} \\
& \quad(2.6)
\end{aligned}
$$

where

$$
\begin{aligned}
& c_{5}(j, \mu)=\int_{0}^{\frac{1}{2}}|\tau(\mu-\tau)|^{j} d \tau=\int_{0}^{\mu} \tau^{j}(\mu-\tau)^{j} d \tau \\
& +\int_{\mu}^{\frac{1}{2}} \tau^{j}(\tau-\mu)^{j} d \tau=\frac{(1-2 \mu)^{j+1}}{4^{j+1}(j+1)(1-\mu)}, \\
& c_{6}(j, \mu)=\int_{\frac{1}{2}}^{1}|(1-\tau)(\lambda-\tau)|^{j} d \tau=\int_{\frac{1}{2}}^{\lambda}(1-\tau)^{j}(\tau-\lambda)^{j} d \tau \\
& \quad+\int_{\lambda}^{1}(1-\tau)^{j}(\tau-\lambda)^{j} d \tau=\frac{(2 \lambda-1)^{j+1}}{4^{j+1}(j+1) \lambda} .
\end{aligned}
$$

Springer 
In order to calculate $c_{7}^{\frac{1}{k}}(k, p, q)$ and $c_{8}^{\frac{1}{k}}(k, p, q)$ using substitution $\xi=p^{2(1-\tau)} q^{2 \tau}$, it leads to

$c_{7}(k, p, q)=\int_{0}^{\frac{1}{2}}\left(p^{2(1-\tau)} q^{2 \tau}\right)^{k} d \tau=\frac{p^{k}}{2} \mathrm{~L}\left(p^{k}, q^{k}\right)$,

$c_{8}(k, p, q)=\int_{\frac{1}{2}}^{1}\left(p^{2(1-\tau)} q^{2 \tau}\right)^{k} d \tau=\frac{q^{k}}{2} \mathrm{~L}\left(p^{k}, q^{k}\right)$.

Hence, (2.5) easily found from (2.7).

Corollary 2 A function $f: I \subseteq \mathbb{R}^{+} \rightarrow \mathbb{R}$ be a twice differentiable function on $I^{o}$ such that $f^{\prime \prime} \in L^{1}([p, q])$, where $p, q \in I$ with $p<q$. If $\left|f^{\prime \prime}\right|^{k}$ is quasi-geometrically convex on $[p, q]$ for some fixed conjugate numbers $j, k \geq 0$ with $k>1$ and for $l, m \in \mathbb{R}$ with $l<m$, then the following inequality holds

$$
\begin{aligned}
& \left|m_{f}\left(\frac{l}{m}, p, q\right)\right| \leq\left(\ln \left(\frac{q}{p}\right)\right)^{2} \frac{1}{m^{j}(j+1)} \\
& \quad \times\left[\frac{(m-2 l)^{j+1}}{4^{j+1}(m-l)}\right]\left(\sup \left\{\left|f^{\prime \prime}(p)\right|^{k},\left|f^{\prime \prime}(q)\right|^{k}\right\}\right)^{\frac{1}{k}} \\
& \quad \times\left\{c_{7}^{\frac{1}{k}}(k, p, q)+c_{8}^{\frac{1}{k}}(k, p, q)\right\},
\end{aligned}
$$

where

$$
\begin{aligned}
& c_{5}\left(j, \frac{l}{m}\right)=c_{6}\left(j, \frac{l}{m}\right)=\frac{1}{m^{j}(j+1)}\left[\frac{(m-2 l)^{j+1}}{4^{j+1}(m-l)}\right], \\
& c_{7}(k, p, q)=\frac{p^{k}}{2} \mathrm{~L}\left(p^{k}, q^{k}\right), c_{8}(k, p, q)=\frac{q^{k}}{2} \mathrm{~L}\left(p^{k}, q^{k}\right),
\end{aligned}
$$

and $m_{f}\left(\frac{l}{m}, p, q\right)$ fixed in Corollary 1

Proof Proof is exchangeable with Theorem 2 using substitution $\lambda=1-\frac{l}{m}$ and $\mu=\frac{l}{m}$.

Theorem 3 A function $f: I \subseteq \mathbb{R}^{+} \rightarrow \mathbb{R}$ be a twice differentiable function on $I^{o}$ such that $f^{\prime \prime} \in L^{1}([p, q])$, where $p, q \in I$ with $p<q$. If $\left|f^{\prime \prime}\right|^{k}$ is quasi-geometrically convex on $[p, q]$ for some fixed conjugate numbers $j, k \geq 0$ where $k>1$ and $0 \leq \mu \leq 1 / 2 \leq \lambda \leq 1$, then the following inequality holds

$\left|M_{f}(\lambda, \mu, p, q)\right| \leq\left(\ln \left(\frac{q}{p}\right)\right)^{2}\left(\sup \left\{\left|f^{\prime \prime}(p)\right|^{k},\left|f^{\prime \prime}(q)\right|^{k}\right\}\right)^{\frac{1}{k}}$ $\times\left\{c_{7}^{\frac{1}{j}}(j, p, q) c_{5}^{\frac{1}{k}}(k, \mu)+c_{8}^{\frac{1}{j}}(j, p, q) c_{6}^{\frac{1}{k}}(k, \lambda)\right\}$, where

$c_{5}(k, \mu)=\frac{(1-2 \mu)^{k+1}}{4^{k+1}(k+1)(1-\mu)}, c_{6}(k, \lambda)=\frac{(2 \lambda-1)^{k+1}}{4^{k+1}(k+1) \lambda}$,

$c_{7}(j, p, q)=\frac{p^{j}}{2} \mathrm{~L}\left(p^{j}, q^{j}\right), c_{8}(j, p, q)=\frac{q^{j}}{2} \mathrm{~L}\left(p^{j}, q^{j}\right)$,

such that $\frac{1}{j}+\frac{1}{k}=1$.

Proof From Lemma 2 by applying Hölder inequality and using the quasi-geometrically convexity on $[p, q]$ of $\left|f^{\prime \prime}\right|^{k}$, we have

$$
\begin{aligned}
& \left|M_{f}(\lambda, \mu, p, q)\right|=\left(\ln \left(\frac{q}{p}\right)\right)^{2} \\
& \quad \times\left\{( \int _ { 0 } ^ { \frac { 1 } { 2 } } ( p ^ { 2 ( 1 - \tau ) } q ^ { 2 \tau } ) ^ { j } d \tau ) ^ { \frac { 1 } { j } } \left(\int_{0}^{\frac{1}{2}}|\tau(\mu-\tau)|^{k}\right.\right. \\
& \left.\quad \times \sup \left\{\left|f^{\prime \prime}(p)\right|^{k},\left|f^{\prime \prime}(q)\right|^{k}\right\} d \tau\right)^{\frac{1}{k}} \\
& \quad+\left(\int_{\frac{1}{2}}^{1}\left(p^{2(1-\tau)} q^{2 \tau}\right)^{j} d \tau\right)^{\frac{1}{j}}\left(\int_{\frac{1}{2}}^{1}|(1-\tau)(\tau-\lambda)|^{k}\right. \\
& \left.\left.\quad \times \sup \left\{\left|f^{\prime \prime}(p)\right|^{k},\left|f^{\prime \prime}(q)\right|^{k}\right\} d \tau\right)^{\frac{1}{k}}\right\}
\end{aligned}
$$

$$
\begin{aligned}
& \left|M_{f}(\lambda, \mu, p, q)\right|=\left(\ln \left(\frac{q}{p}\right)\right)^{2} \\
& \quad \times\left(\sup \left\{\left|f^{\prime \prime}(p)\right|^{k},\left|f^{\prime \prime}(q)\right|^{k}\right\}\right)^{\frac{1}{k}} \\
& \quad\left\{\left(\int_{0}^{\frac{1}{2}}|\tau(\mu-\tau)|^{k} d \tau\right)^{\frac{1}{k}}\right. \\
& \quad \times\left(\int_{0}^{\frac{1}{2}}\left(a^{2(1-\tau)} q^{2 \tau}\right)^{j} d \tau\right)^{\frac{1}{j}}+\left(\int_{\frac{1}{2}}^{1}|(1-\tau)(\tau-\lambda)|^{k} d \tau\right)^{\frac{1}{k}} \\
& \left.\quad\left(\int_{\frac{1}{2}}^{1}\left(p^{2(1-\tau)} q^{2 \tau}\right)^{j} d \tau\right)^{\frac{1}{j}}\right\}
\end{aligned}
$$

where

$$
\begin{aligned}
& c_{5}(k, \mu)=\int_{0}^{\frac{1}{2}}|\tau(\mu-\tau)|^{k} d \tau=\left[\frac{(1-2 \mu)^{k+1}}{4^{k+1}(k+1)(1-\mu)}\right], \\
& c_{6}(j, \lambda)=\int_{\frac{1}{2}}^{1}|(1-\tau)(\lambda-\tau)|^{k} d \tau=\frac{(2 \lambda-1)^{k+1}}{4^{k+1}(k+1) \lambda} .
\end{aligned}
$$


In order to calculate $c_{7}^{\frac{1}{k}}(k, p, q)$ and $c_{8}^{\frac{1}{k}}(k, p, q)$ using $\xi=p^{2(1-\tau)} q^{2 \tau}$, it leads to

$c_{7}(j, p, q)=\int_{0}^{\frac{1}{2}}\left(p^{2(1-\tau)} q^{2 \tau}\right)^{j} d \tau=\frac{p^{j}}{2} \mathrm{~L}\left(p^{j}, q^{j}\right)$,
$c_{8}(j, p, q)=\int_{\frac{1}{2}}^{1}\left(p^{2(1-\tau)} q^{2 \tau}\right)^{j} d \tau=\frac{q^{j}}{2} \mathrm{~L}\left(p^{j}, q^{j}\right)$.

Hence, (2.9) easily found from (2.11).

Corollary 3 A function $f: I \subseteq \mathbb{R}^{+} \rightarrow \mathbb{R}$ be a twice differentiable function on $I^{o}$ such that $f^{\prime \prime} \in L^{1}([p, q])$, where $p, q \in I$ with $p<q$. If $\left|f^{\prime \prime}\right|^{k}$ is quasi-geometrically convex on $[p, q]$ for some fixed conjugate numbers $j, k \geq 0$ and for $l, m \in \mathbb{R}$ with $l<m$, then the following inequality holds

$$
\begin{aligned}
\mid m_{f} & \left(\frac{l}{m}, p, q\right) \mid \leq\left(\ln \left(\frac{q}{p}\right)\right)^{2} \frac{1}{m^{k}(k+1)} \\
& \times\left[\frac{(m-2 l)^{k+1}}{4^{k+1}(m-l)}\right]\left(\sup \left\{\left|f^{\prime \prime}(p)\right|^{k},\left|f^{\prime \prime}(q)\right|^{k}\right\}\right)^{\frac{1}{k}} \\
& \times\left\{c_{7}^{\frac{1}{j}}(j, p, q)+c_{8}^{\frac{1}{j}}(j, p, q)\right\},
\end{aligned}
$$

where

$$
\begin{aligned}
& c_{5}\left(k, \frac{l}{m}\right)=c_{6}\left(k, \frac{l}{m}\right)=\frac{1}{m^{k}(k+1)}\left[\frac{(m-2 l)^{k+1}}{4^{k+1}(m-l)}\right], \\
& \times c_{7}(j, p, q)=\frac{a^{p}}{2} \mathrm{~L}\left(p^{j}, q^{j}\right), c_{8}(j, p, q)=\frac{q^{j}}{2} \mathrm{~L}\left(p^{j}, q^{j}\right),
\end{aligned}
$$

and $m_{f}\left(\frac{l}{m}, p, q\right)$ fixed in Corollary 1 .

Proof Proof is exchangeable with Theorem 3 using substitution $\lambda=1-\frac{l}{m}$ and $\mu=\frac{l}{m}$.

\section{Concluding remarks}

Some results have been developed by generalizing both Hadamard's and Simpson's inequalities for quasi-geometrically convex mapping by defining a new identity for twice differentiable mappings. It is also to be mentioned that the proofs of the corollaries 1,2 and 3 lead to respective Theorems 1, 2 and 3 using the substitution $\lambda=1-\frac{l}{m}$ and $\mu=\frac{l}{m}$.

\section{Compliance with ethical standards}

Conflict of interest The authors declare that they have no conflict of interests.

Open Access This article is distributed under the terms of the Creative Commons Attribution 4.0 International License (http://creativecommons.org/licenses/by/4.0/), which permits unrestricted use, distribution, and reproduction in any medium, provided you give appropriate credit to the original author(s) and the source, provide a link to the Creative Commons license, and indicate if changes were made.

\section{References}

1. Niculescu, C.P., Persson, L.E.: Convex functions and their Applications: A Contemporary Approach. CMS Books in Mathematics, vol. 23. Springer, New York (2006)

2. Zhang, Tian-Yu., Qi, Feng: Integral inequalities of Hermite-Hadamard type for m-AH convex functions. Turk. J. Anal. Number Theory 2(3), 60-64 (2014)

3. Özdemir, M.E.: Some inequalities for the s-Godunora-Levin type functions. Math. Sci. 9(1), 27-32 (2015)

4. Barani, A., Malmir, F.: Hermite-Hadamard inequality for geometrically qausi convex functions on a rectangular box. Math. Sci. Lett. 5(1), 59-69 (2016)

5. Mo, H., Sui, X.: Hermite-Hadamard type inequalities for generalized s-convex functions on real line fractal set $\mathbb{R}^{\alpha}(0<\alpha<1)$. Math. Sci. 11(3), 241-246 (2017)

6. Akdemir, A.O., Özdemir, M.E., Set, E.: New Hadamard-type inequalities for $\mathrm{m}$-convex and $(\mathrm{a}, \mathrm{m})$-convex functions. Math. Sci. Lett. 7(3), 133-138 (2018)

7. Özdemir, M.E.: Inequalities on geometrically convex functions. Math. Sci. Lett. 7(3), 145-149 (2018)

8. Niculescu, C.P.: Convexity according to the geometric mean. Math. Inequal. Appl. 3(2), 155-167 (2000)

9. Niculescu, C.P.: Convexity according to means. Math. Inequal. Appl. 6(4), 571-579 (2003)

10. İşcan, İ.: New general integral inequalities for quasi-geometrically convex functions via fractional integrals. J. Inequal. Appl. 491, 15 (2013)

11. İşcan, İ: Some new Hermite-Hadamard type inequalities for geometrically convex functions. Math. Stat. 1(2), 86-91 (2013)

12. İşcan, İ.: Some generalized Hermite-Hadamard type inequalities for quasi-geometrically convex functions. Am. J. Math. Anal. 1(3), 48-52 (2013)

13. İşcan, İ., Kerim, B., Selim, N.: Hermite-Hadamard and Simpson type inequalities for differentiable quasi-geometrically convex functions. Turk. J. Anal. Number Theory 2(2), 42-46 (2014)

Publisher's Note Springer Nature remains neutral with regard to jurisdictional claims in published maps and institutional affiliations. 